\title{
Deterioration Prediction Modelling and Inspection Schedule Estimation for Concrete Bridge Decks
}

\author{
Aqeed Mohsin Chyad ${ }^{1}$, Osama Abudayyeh ${ }^{2}$ \\ 1. Dept. of Civil and Construction Engineering, Babylon University, Hillah, Babel, Iraq \\ 2. Dept. of Civil and Construction Engineering, Western Michigan Univ., Kalamazoo, MI 49008, USA \\ E-mail: aqeedmohsinch.chyad@wmich.edu (corresponding author); Osama.abudayyeh@wmich.edu
}

Received: 7 January 2020; Accepted: 13 February 2020; Available online: 31 March 2020

\begin{abstract}
Accurate and reliable deterioration rate estimates for concrete bridge decks are an important part of the overall bridge condition assessment. The main objective of this paper is to determine the time in condition ratings (TICRs) of concrete bridge decks and assess the impact of average daily traffic (ADT), age, and deck area on the bridge deck condition. Condition ratings of bridge decks over 24 years for Michigan state were collected from the National Bridge Inventory (NBI) data. The Anderson-Darling statistical test was used to evaluate and rank five practical probability distribution functions to select the best fit for Michigan state data. The results indicate that the best statistical model for Michigan state data is the lognormal function. It was illustrated that the TICR decreases when the condition rating decreases. When a concrete bridge deck condition is rated at 8 , it can take 11.29 years to drop to the lower rating of 7 . However, when the concrete bridge deck condition is rated at 4 , it may take 6.64 years to drop to the lower condition rating of 3. It was also observed that on average, bridge decks in Michigan stay much longer than the typical inspection interval (i.e., 2 years), suggesting that inspection intervals can be longer than 2 years for bridges in good condition ranges. The results also show that ADT, age, and deck area are important factors in the deterioration rates of concrete bridge decks.
\end{abstract}

Keywords: Federal Highway Administration (FHWA); Time in condition ratings (TICRs); Average daily traffic (ADT); National Bridge Inventory (NBI).

\section{Introduction}

National Bridge Inspection Standards (NBISs) were established by the Federal-Aid Highway Act of 1968 and the Surface Transportation Assistance Act (STAA) of 1978 directly after the collapse of the Ohio River Bridge (also known as the Silver Bridge) in 1967 [1]. These standards describe the requirements for regular periodical inspection of bridges. The standards also outline the professional qualifications necessary to serve as an inspector.

Concrete decks are among the most susceptible parts of bridges and their service lives are typically shorter than those of other components because they are exposed to deterioration produced by direct contact with traffic and other environmental factors such as freeze/thaw cycles or deicing materials in cold weather regions [2, 3]. Basically, the resources needed for rehabilitation, replacement, and repair of concrete bridge decks are typically inadequate $[1,4]$. The Federal Highway Administration (FHWA), therefore, is continuously working to support scientific and technological research to achieve both short and long-term results for required enhancements [4]. One of the significant issues that face transportation agencies is how to reduce the cost of bridge deck maintenance [5]. Researchers have suggested the use of accurate and reliable condition assessment techniques can assist in reducing the costs and increasing the efficiency of concrete bridge deck maintenance and repair $[5,6]$.

Since routine inspection requirements were first established in the early 1970s, the regular two-year interval assigned by the NBIS has been effective in guaranteeing an acceptable level of protection and serviceability for highway bridges [7]. Currently, regardless of condition rating, most bridges in the United States are scheduled for inspection at a uniform calendar interval of two years. However, because of the fixed two-year inspection schedule of both newly constructed bridges with little or no deterioration along with old bridges with more deteriorated components, inefficiencies in the allocation of inspection resources are observed [8].

The fixed inspection intervals (i.e., the time between two consecutive inspections) can be reduced or increased based on conditions established by the bridge owner for bridges in certain condition rating ranges. Inspection intervals of up to six years can be acceptable for certain bridges that meet condition criteria. Commonly, bridges with low ADT and short spans that are in good condition can be qualified for the extension of inspection intervals $[8,9]$. An earlier study used statistical models to analyse Oregon bridge condition data extracted from the National 
Bridge Inspection Standard (NBIS) [10]. The authors concluded that bridges with good condition ratings tend to stay in those ratings longer than two years and therefore suggested a possible extension of the inspection intervals.

\subsection{Condition assessment of concrete bridge decks and causes of deterioration}

Maintaining the safety and serviceability of concrete bridge decks is very important because bridges are a critical part of the transportation networks. FHWA has developed condition assessment processes for all bridges in the United States where they undergo inspection every two years based on NBISs to determine the condition ratings for each major element in a bridge [11]. In contrast, the inspection intervals in Europe may reach up to five or six years based on inspector qualifications and experience, and can go as long as nine years as in the case of France [12]. Mathematical and statistical models have been developed to assess and predict the condition of bridge elements using the NBI database [13]. Developing optimum prediction models is crucial for making maintenance, repair, or replacement decisions.

According to the NBI, there are more than 600,000 bridges in service around the United States. Half of these bridges were constructed before the year 1970, and 25\% of the total bridges require rehabilitation, repair, and/or reconstruction. The available resources compared to the enormous quantity of work required for accomplishing the repair, rehabilitation, and reconstruction are often very limited [1]. Based on the reports available from the FHWA, more than 100 million $\mathrm{m}^{2}$ of the entire 360 million $\mathrm{m}^{2}$ U.S. concrete bridges are either structurally deteriorated (SD) or functionally obsolete (FO). The decision to rehabilitate or replace a bridge is influenced by technical and economic factors. A comprehensive inspection is needed for all types of bridges to provide appropriate information about the general condition of the bridges. This information includes measurements and the accompanying defects that are discovered in bridges under examination. Once the inspection phase is completed, analysis of collected data commences. These data are used to determine the best maintenance or rehabilitation method to maintain the service life of the bridge [14].

Deterioration of concrete bridge decks occurs due to many reasons. Therefore, it is very important to study the most expected factors impacting on a bridge condition [15]. Old bridges may deteriorate faster than newer ones $[16,17]$. Additionally, as the concrete bridge deck is the main component that provides the riding surface, it is exposed to deterioration more than the other parts of a bridge, making ADT as an important factor for consideration [18]. Furthermore, large areas of bridge decks can be exposed to random types of defects that may result in inaccurate condition rating [6]. For example, if a relatively small part of a large area of a bridge deck has a defect level matching to a rating of 5 , an inspector may rate the entire deck as 5 , possibly resulting in a non-optimal maintenance decision. In this study, the impact of these three factors (i.e., ADT, age, and deck area) on the deterioration rate will be investigated to help in developing future deterioration prediction models.

Currently, there are several methods that have been developed for bridge condition assessment, including the fuzzy based analytic hierarchy approach [19], deterministic methods [17], probability distribution methods [6,10], and Markov chain models [13]. These methods can be used to measure the deterioration rates of bridges. Consequently, an enhancement in the prediction of the remaining service life of bridges has resulted due to using these methods.

\subsection{Condition ratings of concrete bridge decks}

The NBI database includes condition ratings for the major bridge elements, including the deck, superstructure, and substructure for a period of 24 years (i.e., from 1992 to 2015) (Federal Highway Administration [20,21]. According to NBIS, condition ratings for each major element in a bridge varies from 0 to 9 . Typically, a bridge is considered structurally deficient if the deck receives a condition rating of 3 or less, while a condition rating of 7 or more is very desirable [22]. Overall, these condition ratings are indicators of the level of bridge deck performance and required maintenance actions [23].

The condition ratings of bridges typically decrease over time due to the deteriorations that occur in the major elements. In other words, the major elements are rated at 9 when bridges are newly constructed. They stay in the same condition rating for a while and then start to deteriorate with time and drop down to the next condition rating. This process is repeated again, and the condition rating drops further [17]. In this study, the change in the condition rating over time will be investigated in an attempt to identify and model deterioration trends to develop prediction models.

\section{Goals and significance}

The goal of the study described in this paper is to estimate the TICRs in each condition rating of Michigan bridge decks and investigate the impact of ADT, age, and deck area on bridge condition and on the inspection intervals. A concrete bridge deck is typically the primary load path, and its condition is a significant aspect of the integrity and serviceability of the bridge [14]. In Michigan, there are around more than 10,000 bridges that require inspection every two years. The objective is to develop condition assessment schedules for bridge decks that 
address the maintenance needs of the more critical bridges within the resource constraints of transportation agencies. The study described in this paper will analyse condition data of Michigan bridges available through NBI to determine how many years, on average, a bridge deck remains in a certain condition rating and to evaluate the impact of ADT, age, and deck area on the deterioration rates of bridge decks. Additionally, condition ratings data over a 24 year were analysed under the Anderson darling test to determine the best statistical model that can represent Michigan state data.

\section{Data sources and handling}

Two main issues will be explored and discussed in this study: 1) The data needed to track bridge deterioration rates, and 2) Factors that affect bridge deterioration rates. NBI condition ratings are used to rank bridges and can be used to track concrete bridge deck deterioration rates [20,21]. Data from 1992 to 2015 was used in this study. Since inspections are performed yearly or biennially, NBI records of condition ratings are available for each of the main bridge elements. These condition ratings are then transformed into consistent NBI condition codes, which are also identified by FHWA.

\subsection{Data collection methods}

The inspection and maintenance of bridges have become a priority for U.S. departments of transportation. Currently, visual inspection and chain drag are the main methods to inspect bridge decks and can detect cracks, spalls, and delaminations. They are essentially the main technique used to collect data about bridge condition. However, they are considered subjective and ambiguous because they depend on the experience of the inspector, definition of the deteriorations, the condition level of the defects, and other factors. Non-destructive testing methods are beginning to gain acceptance, but are still under research. Many studies have been conducted to determine if non-destructive techniques are adequate for bridge inspection. Some of these non-destructive techniques include Ground Penetration Radar (GPR), Impact Echo, and infrared thermal imaging [3, 24-27].

\subsection{Data pre-processing}

For an accurate evaluation of deterioration rates, inspection data must be treated to eliminate the effects of issues other than uniform maintenance that may result in an increase or decrease in condition ratings. These issues include repair, miscoding [17]. This study is based on the NBI database, which includes numerical ratings of bridge decks for the period of 1992 to 2015. The treatment of data was performed in several steps:

1) Due to the fact that ratings of $0-3$ are considered severe conditions that require immediate attention, such bridges typically undergo significant rehabilitation or replacement rather than staying in their current condition. Therefore, these condition ratings were not included in this study. It is also worth noting that there are few bridges with condition ratings of $0-3$ as compared with other condition ratings. Additionally, data with condition ratings of 9 were removed from the analysis because they represent new bridge construction with no deterioration [10].

2) Data with condition ratings of $N$ (for not applicable data) and inspection data with unusual rating drop were removed from the data set. For example, if the bridge was in the condition rating of 8 for 3 years and then dropped to a condition rating of 6 or 5 for 2 years and then jumped to a condition rating of 7 or 8 for 2 or 3 years and this process was replicated every 2 or 3 years for the period from 1992 to 2015 without any records about rehabilitation or repair, then these data were eliminated [1].

3) Bridges that did not have key parameter records such as deck rating, year built, ADT, deck width, and structure length were eliminated [17].

4) All bridges that had recently undergone rehabilitation, repair, or reconstruction of the deck were clipped [6].

5) If the time in condition rating (TICR) was 3 years or less for sequential inspection cycles at the beginning or the end of the study period interval (1992-2015), data were clipped from the original record. For example, if the bridge was in the condition rating of 6 in the 1992 and 1994 (which is 3 years (TICR)) and then dropped to a condition rating of 5 or jumped to a condition rating of 7 due to rehabilitation or repair in the next inspection cycle in 1996, then the data from 1992 to 1994 were removed. Similarly, if the condition rating of the bridge changed to 6 in 2013 and stayed at 6 through 2015, this data was also clipped. The 3-year threshold for clipping was based on a sensitivity analysis of different probable trimming values ranging from 3 to 7 years. The results showed that TICR of more than 3 years at the beginning or end of the available data set had the largest impact on the final analysis results. Consequently, 3 years was selected as a suitable value. A similar analysis was performed on the Oregon data set that resulted in a 5-year clipping threshold, suggesting that this criterion would depend on the data set and the time interval under investigation [10].

6) Some of the NBI records for the concrete bridge deck showed an increase or decrease in the condition rating for 1 or 2 years, and then a return to the same condition rating without any recognized rehabilitation. These increases and decreases are considered inspector errors and the condition rating is manually revised to be identical to the previous value. For example, if the concrete bridge deck was at condition rating of 7 for 5 years, then 
dropped to a condition rating of 6 for 2 years, and then returned to the initial value of 7 for 4 years, we would consider this as an error and would correct the results to display the concrete bridge deck at condition 7 for 11 years $[10,16]$.

\subsection{Assumptions}

A few assumptions were made to simplify the analysis as follows:

1) Only bridges with concrete decks were included in the analysis (item 107 in NBI data).

2) The original construction date of the bridge is determined from the year built (item 27 in NBI data). This is used as the base when determining if the overlay and the deck are part of the original construction.

3) The value of ADT is determined from the total ADT (item 29 in NBI data). Bridges with ADT of 0 were eliminated from the data set and the data of this factor were divided into three categories: less than 4,000, 4,000 to10,000, and more than 10,000 (vehicles/day).

4) The age of the deck is determined by comparing the current year to the year built (item 27 in NBI data). Then, the year-built data for bridge decks have been updated with the new dates of reconstruction as listed in the NBI (item 106) when bridges have been reconstructed.

5) The deck areas were calculated from the structure length (item 49 in NBI data) and the deck width (item 51 in NBI data).

6) The age values in years were classified into two ranges: less than or equal to 25 and more than 25 years. (The lifespan of the most bridges were designed to be 50 years [28]. Therefore, bridges within this range were analysed in this study.

7) The deck area values in square meters $\left(\mathrm{m}^{2}\right)$ were divided into two groups: less than $500 \mathrm{~m}^{2}$ and more than $500 \mathrm{~m}^{2}$.

To verify whether the bins for the above factors are appropriate for the study, Kruskal-Wallis test was performed. This test was chosen because condition rating data are not normally distributed. Therefore, the bins for each factor were analysed under this test to evaluate if there was significant statistical difference between the factor groups. Table 1 shows the parameters of the Kruskal-Wallis test. Statistical significance between bins exists when the pvalue is less than the 0.05 [29]. As shown in the table, all p-values were less than 0.05 , suggesting that all bins were classified appropriately. Additionally, the chi square values further demonstrate that the TICR data vary between bins for each factor and support the appropriateness of the bin selections.

Table 1. Kruskal-Wallis test for factor groups.

\begin{tabular}{|c|c|c|c|c|c|c|c|}
\hline \multirow{4}{*}{ CR } & \multicolumn{6}{|c|}{ Groups } & \multirow{4}{*}{$\begin{array}{l}\text { \# of } \\
\text { Samples } \\
\end{array}$} \\
\hline & \multirow{2}{*}{\multicolumn{2}{|c|}{$\begin{array}{l}\text { ADT (vehicles/day) } \\
\text { ADT }<4,000,4,000-10,000 \text {, and }>10,000\end{array}$}} & \multirow{2}{*}{\multicolumn{2}{|c|}{$\begin{array}{l}\text { Age (years) } \\
\text { Age } \leq 25 \&>25\end{array}$}} & \multirow{2}{*}{\multicolumn{2}{|c|}{$\begin{array}{l}\text { Area }\left(\mathrm{m}^{2}\right) \\
\text { Area } \leq 500 \&>500\end{array}$}} & \\
\hline & & & & & & & \\
\hline & Chi Square & P-value & Chi Square & P-value & Chi Square & P-value & \\
\hline 8 & 74.331 & 0 & 109.951 & 0 & 70.881 & 0 & 1360 \\
\hline 7 & 33.315 & 0 & 17.168 & 0 & 19.214 & 0 & 2321 \\
\hline 6 & 19.04 & 0 & 8.101 & 0.004 & 18.953 & 0 & 1845 \\
\hline 5 & 11.145 & 0.004 & 7.612 & 0.006 & 22.715 & 0 & 760 \\
\hline 4 & 7.774 & 0.021 & 12.085 & 0.001 & 5.202 & 0.023 & 176 \\
\hline
\end{tabular}

\section{Analysis of national bridge inspection condition data}

The study described in this paper is based on the NBI bridge inspection data for the period from 1992 to 2015. In this section, determining the best statistical method for Michigan state will be investigated. Then, the best statistical model for Michigan concrete bridge deck will be used to evaluate the TICRs. Table 2 shows the description of condition ratings for Michigan concrete bridge decks in addition to their ages in 2015.

\subsection{Analysis of NBI condition data for Michigan bridge decks}

In this study, statistical analyses were performed to determine the distribution model that best fits the TICR values for Michigan bridge deck condition rating data. There are three most commonly used goodness-of-fit tests available for evaluating and ranking the most commonly used statistical models: Anderson-Darling (AD), ChiSquare, and Kolmogorov-Smirnov (K-S) [30]. The Anderson-Darling is the most appropriate goodness-of-fit test for the bridge condition data at hand. It is performed to examine data derived from a population with a particular distribution and produces more weight to the tails than does the K-S test. The AD test is also more precise at the tails data than the Chi-Square and K-S tests. Since AD uses the maximum difference between the cumulative distribution function curves, it gives more weight to outliers than KS. Thus, the test considers all the differences at the tail end that may be neglected or removed by the other test methods [31]. The most frequently used statistical 
models under the Anderson-Darling test are the exponential, Weibull, lognormal, normal, and gamma distributions [32]. In this study, these models were investigated.

The Michigan concrete bridge deck condition ratings that were used in this investigation ranged from 4 to 8 over the past 24 years (1992-2015). Table 3 summarizes the Anderson-Darling test values of the five commonly used statistical models. In the table, the statistical analyses are grouped by condition rating values. The lognormal probability distribution function had the smallest Anderson-Darling test value for most of the CR values, making it the best statistical model for the TICR data set. The Gamma distribution function was a better fit than (but fairly close to) the lognormal distribution method for CR values of 6 and 8, but was fairly behind for the other three CR values, resulting in the choice of lognormal as the best fit. It is also clear from Table 3 that the exponential distribution method is the least desirable model with the highest Anderson-Darling test values. Moreover, goodness-of-fit tests were done for each group of factors and the lognormal distribution function was the best distribution for each factor in the Michigan dataset.

Table 2. Condition ratings for Michigan concrete bridge decks, 2015.

\begin{tabular}{llll}
\hline Condition Rating & Description & No. of Bridges & Average Age (year) \\
\hline 9 & Excellent Condition & 229 & 5.67 \\
8 & Very good condition & 1,606 & 18.31 \\
7 & Good condition & 3,272 & 32.25 \\
6 & Satisfactory condition & 2,598 & 43.16 \\
5 & Fair condition & 1,029 & 53.97 \\
4 & Poor condition & 375 & 58.98 \\
3 & Serious condition & 121 & 65.89 \\
2 & Critical condition & 18 & 72.39 \\
1 & Imminent failure condition & 1 & 70 \\
0 & Failed condition & 6 & 102.5 \\
Total & & 9,255 & \\
\hline
\end{tabular}

Table 3. Anderson-Darling test values for TICR statistical models for Michigan concrete bridge decks.

\begin{tabular}{lllllll}
\hline Condition Rating & Normal & Lognormal & Gamma & Weibull & Exponential & \# of Samples \\
\hline 8 & 21.70 & 17.86 & 17.62 & 16.16 & 159.11 & 1360 \\
7 & 74.46 & 28.90 & 32.45 & 60.34 & 238.43 & 2321 \\
6 & 39.67 & 15.82 & 15.25 & 33.16 & 222.36 & 1845 \\
5 & 30.58 & 11.04 & 12.40 & 25.29 & 86.19 & 760 \\
4 & 13.08 & 5.46 & 8.22 & 11.11 & 29.41 & 176 \\
\hline
\end{tabular}

\subsection{Condition prediction modelling for Michigan bridge deck data}

The lognormal distribution function is considered one of the most appropriate and flexible models commonly used to determine failures produced by deterioration processes because of certain features of the lognormal random variable such as the non-negative values and the skewness [33]. The lognormal cumulative distribution function is expressed as [34]:

$$
\mathrm{F}\left(\mathrm{t} / \mu, \sigma^{2}\right)=\frac{1}{\sqrt{\left(2 \pi \sigma^{2}\right) t}} \exp \left(-\frac{(\ln t-\mu)^{2}}{2 \sigma^{2}}\right) \quad \mathrm{t}>0, \sigma>0,-\infty<\mu<+\infty
$$

where $t$ is independent positive random variable. $\sigma$ is the shape parameter and $\exp ^{\mu}$ is the scale parameter. $\sigma, \mu$ can be calculated as

$$
\begin{aligned}
& \sigma=\sqrt{\frac{\sum_{i=1}^{N}\left(\ln \left(t_{i}\right)-\mu\right)^{2}}{N}} \\
& \mu=\frac{\sum_{i=1}^{N} \ln \left(t_{i}\right)}{N}
\end{aligned}
$$

$\mathrm{m}$ is the mean, $\mathrm{V}$ is the variance and $\mathrm{S}$ is the standard deviation. $\mathrm{m}, \mathrm{V}$ can be calculated as

$$
\begin{aligned}
& \mathrm{m}=\exp ^{\mu+\sigma^{2} / 2} \\
& \mathrm{~V}=\left(\exp ^{\sigma^{2}}-1\right) \exp ^{2 \mu+\sigma^{2}}
\end{aligned}
$$


S can be calculated from the equation (4).

$$
\mathrm{V}=\mathrm{S}^{2}
$$

In this study, $\mathrm{t}$ is a variable that represents the time interval in which concrete bridge decks can remain in a condition rating before dropping to a lower one (i.e. TICR). For example, if the concrete bridge deck is changed from a condition rating of 8 to 7 and stays at the new rating for 9 years before dropping to 6 , then the time interval is 9 years in this equation. The shape parameter $\sigma(1 \mathrm{a})$ is always more than 0 . The relationship between the value of $\sigma$ and the skewness of the lognormal distribution is positive. An increase (or decrease) in the value of $\sigma$ increases (or decreases) the skewness of the distribution. Skewness is the scale of the symmetry of the distribution. Additionally, when the value of $\sigma$ is less than 1 , the lognormal distribution becomes very close to the normal distribution model. The scale parameter exp $(\mu)(1 \mathrm{~b})$ represents the average of TICRs of concrete bridge decks [35].

\section{Evaluation of the Michigan bridge deck condition prediction model}

The lognormal distribution method was used to model the deterioration rates of Michigan concrete bridge decks using historic data for the period from 1992 to 2015. The data records of concrete bridge decks are distributed on condition ratings (CRs) from 4 to 8 . The results obtained from this study can be used to predict the time needed for periodical inspection of concrete bridge decks.

The values of the shape parameter for all CRs were less than 1, suggesting that the behaviour of the lognormal distribution model was very close to that of a normal distribution. Table 4 shows the values of this parameter in the lognormal distribution model for the different CRs of Michigan concrete bridge decks. As shown in Table 4, all of the values of the scale parameter (i.e., TICRs) are greater than 2 years (the fixed inspection schedule for bridges according to NBI). The TICR decreases when the condition rating decreases. For example, when a concrete bridge deck condition is rated at 8 , it can take 11.29 years to drop to the lower CR by 7 . However, when the concrete bridge deck condition is rated at 4, it may take 6.64 years to drop to the lower condition rating of 3 . Essentially, concrete bridge decks that are in the same condition (i.e., CRs of 7 and 8) tend to stay longer in good condition as compared to those that are in poor condition (i.e., CRs of 5 or less). Figure 1 shows an example of the lognormal probability density function for the Michigan concrete bridge deck condition rating of 5 .

Table 4. The TICR Lognormal Model Parameters for Michigan Concrete Bridge Decks.

\begin{tabular}{llllll}
\hline \multirow{2}{*}{ Parameters } & \multicolumn{5}{c}{ Condition Ratings } \\
\cline { 2 - 6 } & $\mathbf{C R}=\mathbf{4}$ & $\mathbf{C R}=\mathbf{5}$ & $\mathbf{C R}=\mathbf{6}$ & $\mathbf{C R}=\mathbf{7}$ & $\mathbf{C R}=\mathbf{8}$ \\
\hline Shape Parameter $(\sigma)$ & 0.43 & 0.52 & 0.50 & 0.54 & 0.53 \\
Scale Parameter $\left(\exp ^{\mu}\right)$ & 6.64 & 8.25 & 9.63 & 9.97 & 11.29 \\
Mean $(\mathrm{m})$ & 7.28 & 9.43 & 10.39 & 11.57 & 12.98 \\
Standard Deviation $(\mathrm{S})$ & 3.26 & 5.21 & 5.86 & 6.80 & 7.35 \\
\# of Samples & 176 & 760 & 1845 & 2321 & 1360 \\
\hline
\end{tabular}

Figure 2 shows the probability of deterioration rates of different concrete bridge deck condition ratings based on the cumulative distribution functions for CRs ranging from 4 to 8 . Specifically, the Figure shows the probability of a concrete bridge deck remaining in its condition rating before dropping to a lower CR. For example, there is a $10 \%$ probability that the time that a deck at CR of 4 stays in its condition rating will be less than 3.84 years, which is longer than the 2-year inspection schedule. For the same probability (i.e. 0.1), a CR of 8 will take 5.74 years before dropping to a CR of 7. As another example, consider that there is an interest in estimating how long it will take for a concrete bridge deck to deteriorate from a CR of 8 to a CR of 4 . Looking at the 0.05 probability (the probability of failure 5\%; drop from a certain level to the next low level) in Figure 2, dropping from a CR 8 to CR 4 will take around 16.55 years $(4.74+4.07+4.21+3.53)$. This study, therefore, can support decision-makers as they examine the possibility of changing inspection intervals for concrete bridge decks in Michigan, especially for those that are in good condition.

\section{The impact of factors on the TICR}

The impact of each factor (ADT, age, and deck area) on the TICR was investigated. Since these factors are not independent from each other, the investigation was performed by keeping two factors constant and evaluating the effect of the third factor. The following sections will discuss the impact of each factor on the deterioration rates. 


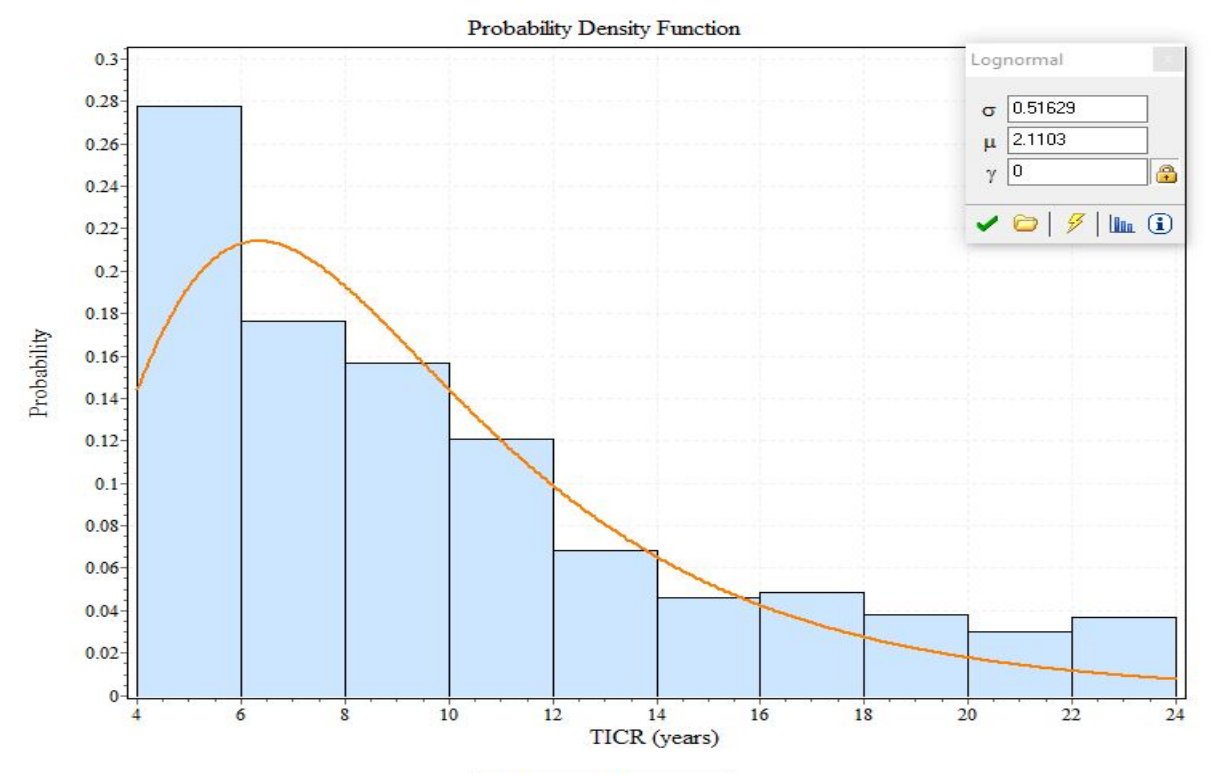

Fig. 1. The Lognormal Probability Density Function for a Condition Rating of 5

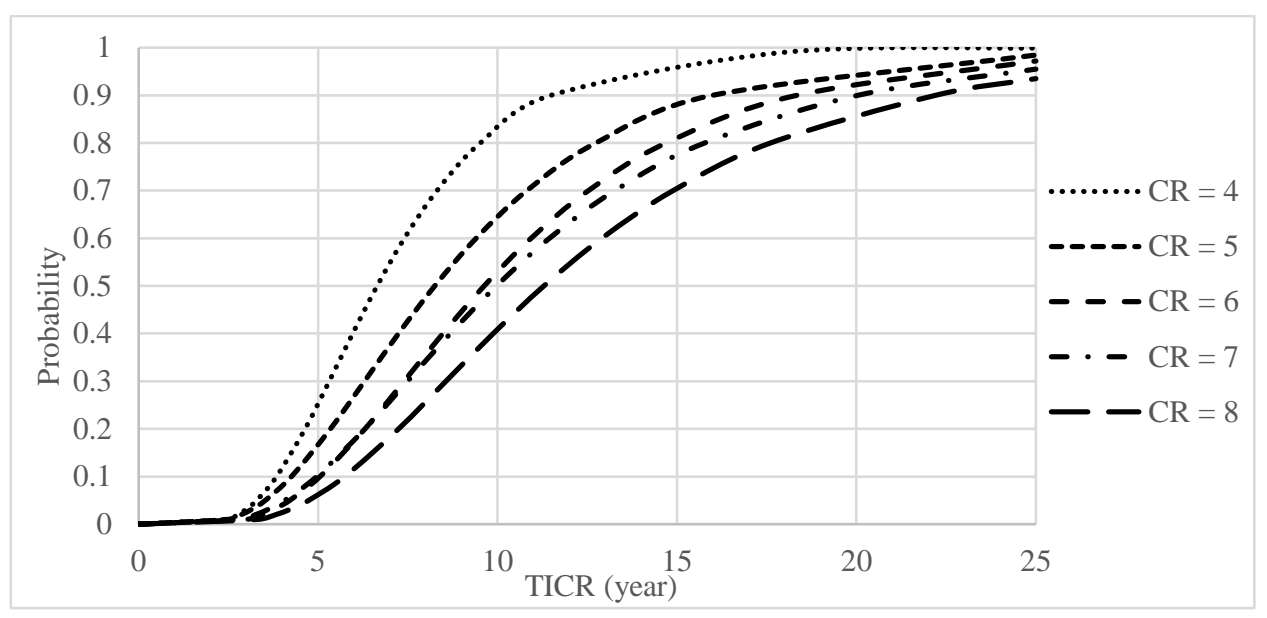

Fig. 2. Cumulative distribution function of concrete bridge decks with different condition ratings.

\subsection{The impact of ADT}

The ADT factor has a major effect on the deterioration rates of concrete bridge decks, as shown in Table 5. Specifically, it was found that ADT can significantly impact a concrete bridge deck when it is still in good condition, and has a slight effect on bridge decks that are in poor condition. For example, the concrete bridge decks in CR of 8 can stay in this condition for 13.35 years before dropping to a condition rate of 7 when the ADT is less than 4,000 vehicles/day with age is less than or equal to 25 years and deck area is less than or equal to $500 \mathrm{~m}^{2}$; but the concrete bridge decks for the same condition rating may just stay 9.68 years before dropping when the ADT is more than 10,000 vehicles/day. This conclusion can be seen in the rest of condition ratings (Table 5).

Previous studies have recommended using the probability of failure (transitioning from one rating to next lower one) that does not exceed 5\%, a risk threshold that can be accepted by transportation authorities (Nasrollahi, \& Washer, 2015). Figure 3 is the 5\% probability chart for TICR under the effect of ADT. This figure, for example, can show how many years it can take for concrete bridge decks to deteriorate from 8 to 4 under the effect of ADT. It may take around 20.49 years to deteriorate from 8 to 4 if the ADT is less than 4,000 vehicles/day with age is less than or equal to 25 years and deck area is less than or equal to $500 \mathrm{~m}^{2}$, while it may take just 16.71 years to deteriorate from 8 to 4 if the ADT is more than 10,000 vehicles/day for the same conditions.

\subsection{The impact of deck age}

The effect of age on the deterioration rates of bridge decks is similar to the effect of ADT. The progress of age showed a significant effect on the deterioration of the bridge decks as shown in Table 5. In fact, with the same 
ADT, age had an effect on the deterioration rate of concrete bridge decks that are in good condition more than those in poor condition. For example, bridge decks within 25 years of service life, ADT is less than 4,000 vehicles/day, and deck area is less than or equal to $500 \mathrm{~m}^{2}$ can stay for 13.35 years in the condition rating of 8 , while for those that are more than 25 years in service for under the same ADT and area may stay just 8.20 years in the condition rating of 8 (see Table 5). This conclusion can be noticed in the other condition ratings (7 to 4 ).

Figure 4 shows that bridges within 25 years, ADT is less than 4,000 vehicles/day, and deck area is less than or equal $500 \mathrm{~m}^{2}$ will have a period of 20.49 years to deteriorate from a condition rating of 8 to 4 , while it can just take 16.9 years for bridges more than 25 years of service with the same conditions under the probability of failure $5 \%$.

Table 5. TICRs (Scale parameter values) with Different Ranges of ADT (vehicles/day), Age (year), and Deck Area $\left(\mathrm{m}^{2}\right)$

\begin{tabular}{lllllll}
\hline \multirow{2}{*}{ Ranges of Other Factors } & Ranges of Certain & \multicolumn{5}{l}{ Time in Condition Ratings (TICR) } \\
\cline { 2 - 6 } & Factor & CR $=\mathbf{4}$ & $\mathbf{C R}=\mathbf{5}$ & $\mathbf{C R}=\mathbf{6}$ & $\mathbf{C R}=\mathbf{7}$ & $\mathbf{C R}=\mathbf{8}$ \\
\hline Age $<25$ years and Deck & ADT $<4,000$ & 7.40 & 8.81 & 10.23 & 10.58 & 13.35 \\
Area $<500 \mathrm{~m}^{2}$ & ADT 4,000-10,000 & 7.20 & 7.89 & 8.92 & 10.34 & 10.52 \\
& ADT $>10,000$ & 6.52 & 7.62 & 8.44 & 9.94 & 9.68 \\
Age $>25$ years and Deck & ADT $<4,000$ & 6.83 & 8.23 & 10.02 & 9.89 & 8.2 \\
Area $<500 \mathrm{~m}^{2}$ & ADT 4,000-10,000 & 6.52 & 751 & 9.79 & 8.41 & 7.66 \\
& ADT $>10,000$ & 5.48 & 7.07 & 9.67 & 7.87 & 7.74 \\
ADT $<4,000$ vehicle/day & Age $<25$ & 7.40 & 8.81 & 10.23 & 10.58 & 13.35 \\
and Area $<500 \mathrm{~m}^{2}$ & Age $>25$ & 6.83 & 8.23 & 10.02 & 9.89 & 8.20 \\
ADT $>10,000$ vehicle/day & Age $<25$ & 6.52 & 7.62 & 8.44 & 9.94 & 9.68 \\
and Area $<500 \mathrm{~m}^{2}$ & Age $>25$ & 5.48 & 7.07 & 9.67 & 7.87 & 7.74 \\
ADT $<4,000$ vehicle/day & Area $<500$ & 7.40 & 8.81 & 10.23 & 10.58 & 13.35 \\
and Age $<25$ years & Area $>500$ & 6.15 & 8.27 & 8.87 & 10.12 & 9.78 \\
ADT $>10,000$ vehicle/day & Area $<500$ & 6.52 & 7.62 & 8.44 & 9.94 & 9.68 \\
and Age $<25$ years & Area $>500$ & 6.12 & 7.02 & 8.08 & 9.08 & 7.66 \\
\hline
\end{tabular}

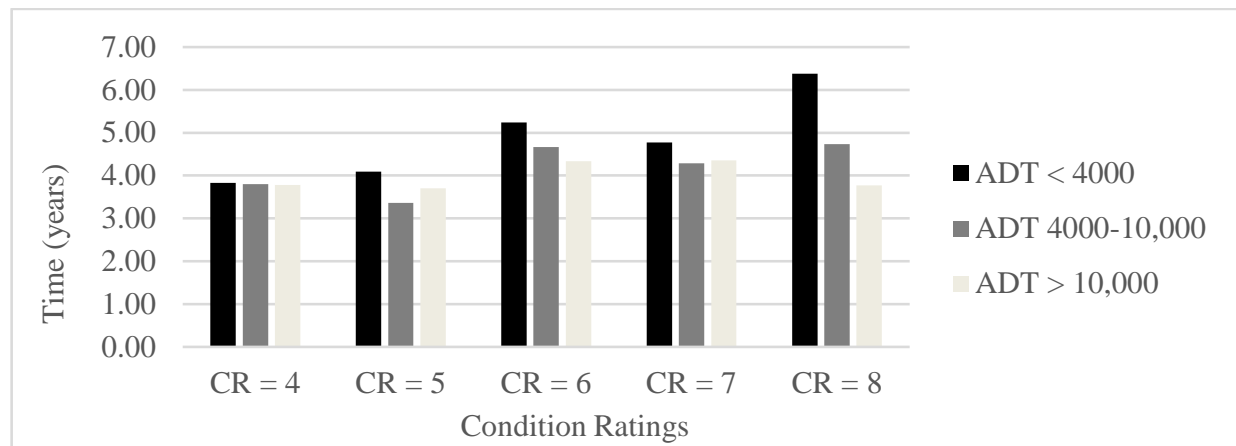

Fig. 3. TICRs for a 5\% probability of failure due to effect of ADT with Age $\leq 25$ (year) and deck area $\leq 500\left(\mathrm{~m}^{2}\right)$.

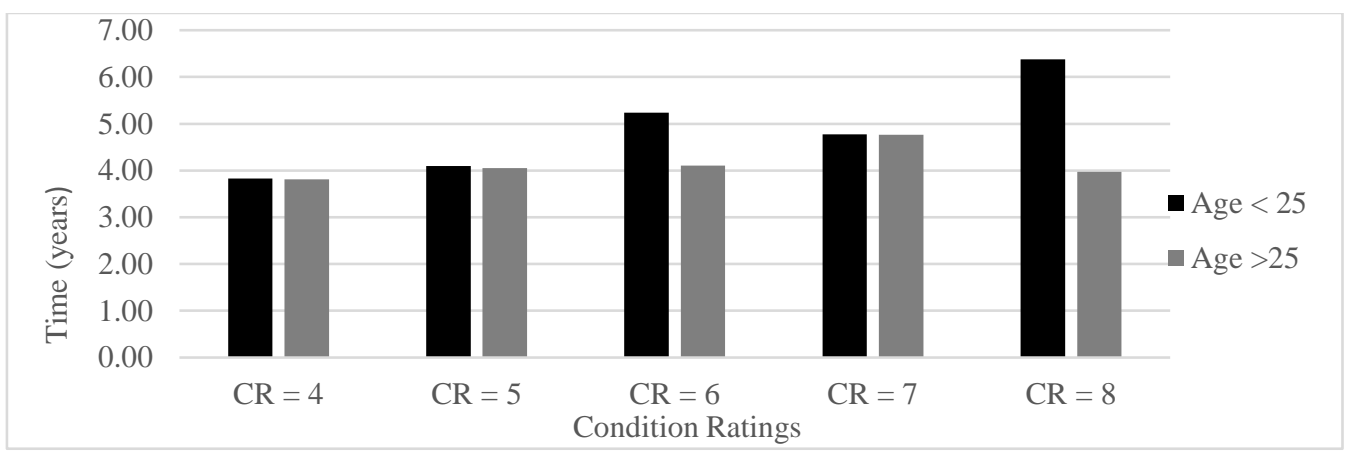

Fig. 4. TICRs for a 5\% Probability of Failure Due to Effect of Age with ADT < 4,000 (vehicle/day) and Deck Area $\leq 500\left(\mathrm{~m}^{2}\right)$. 


\subsection{The impact of deck area}

Similar to ADT and age, the size of the bridge deck area can have a considerable effect on the deterioration rate for all condition ratings of concrete bridge deck (Table 5). Essentially, there is a direct relationship between the deck area and ADT that causes the deck area to have an impact on the deterioration of concrete bridge decks. For example, bridges with deck area is less than $500 \mathrm{~m}^{2}$ with ADT is less than 4,000 vehicles/day and age is less than or equal to 25 years can stay in the condition rating of 8 for 13.35 years, while bridges with deck areas are more than $500 \mathrm{~m}^{2}$ will stay just 9.78 years. Also, bridges with deck area less than $500 \mathrm{~m}^{2}$ with ADT is more than 10,000 vehicles/day and age is more than 25 years can stay in the condition rating of 8 for 7.74 years (Table 5). The same approach can be seen in all condition ratings (condition rating of 4 to 7 ).

Figure 5 shows the time intervals wherein concrete bridge decks can move from good condition to poor condition (i.e., from a condition rating of 8 to 4 ) under the effect of deck area for the probability of failure $5 \%$. It may take around 20.49 years for a concrete bridge deck to deteriorate from 8 to 4 if the area of the bridge decks is less than or equal to $500 \mathrm{~m}^{2}$ with $\mathrm{ADT}$ is less than 4,000 and age is less than or equal to 25 years, while it may take just 16.79 years to deteriorate from 8 to 4 if the area is more than 500 for the same conditions.

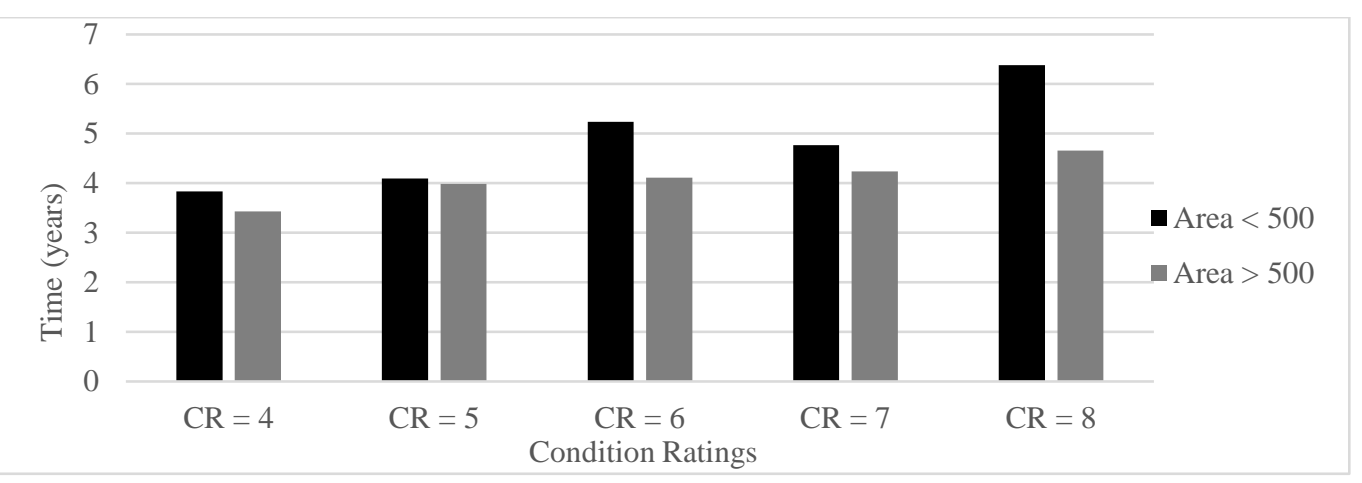

Fig. 5. TICRs for a 5\% Probability of Failure Due to Effect of Area with Age $\leq 25$ (year) and ADT $<4,000$ (vehicles/day).

\section{Concluding remarks}

Bridge condition data from 1992 to 2015 were analysed to determine the TICR of Michigan concrete bridge decks and investigate the impact of ADT, age, and deck area on the deterioration rates of concrete bridge decks. The Anderson-Darling statistical test was used to assess and rate five practical probability distribution methods to choose the best fit for Michigan state in the USA. The results revealed that the lognormal distribution function was the best model for the Michigan data.

This paper illustrated that Michigan concrete bridge decks that are in good condition can stay in those conditions longer than the typical two-year inspection schedules as recommended by the NBISs. Additionally, this study revealed that concrete bridge decks in good conditions deteriorate at a slower rate than those decks in poor conditions. Consequently, inspection schedules for concrete bridge decks can be extended beyond the two years, especially for those decks that are in good condition (CRs of 7, and 8). or for those that are recently constructed. However, if such an action is to be adopted, it must initially be at a slow rate and be carefully monitored before fully extending inspection schedules to ensure bridge safety and to guarantee that proper and timely maintenance actions are not compromised. Essentially, while inspection intervals can be extended up to 6 years in Michigan, which parallels the conclusions of others, this study revealed that there is currently no universal statistical prediction model that can be developed in one state and used by others. However, using the probability of failure $5 \%$ has reduced the extension of inspection intervals up to 4 years. Thus, more studies are required to select the acceptable threshold for this purpose to support decision-makers as they study the possibility of extending inspection intervals.

The results in this paper demonstrated that the ADT, age, and deck area factors have significant impact on the deterioration rates of concrete bridge decks. Future studies are needed to further evaluate each of these factors in more details. For example, more bins for ADT, age, and deck area can be established to provide a more detailed impact analysis for the deterioration rates of concrete bridge decks.

\section{References}

[1] Agrawal AK, Kawaguchi A, Chen Z. Deterioration rates of typical bridge elements in New York. Journal of Bridge Engineering. 2010;15(4):419-429. 
[2] Oh T, Kee SH, Arndt RW, Popovics JS, Zhu J. Comparison of NDT methods for assessment of a concrete bridge deck. Journal of Engineering Mechanics. 2013;139(3):305-314.

[3] Yehia S, Abudayyeh O, Nabulsi S, Abdel-qader I. Detection of common defects in concrete bridge decks using nondestructive evaluation techniques. Journal of Bridge Engineering. 2007;12(2):215-225.

[4] Deza U. Development, evaluation and implementation of sensor techniques for bridges critical to the national transportation system. [Doctoral dissertation]. Iowa State University, Ames, Iowa. 2011.

[5] Scott M, Rezaizadeh A, Delahaza A, Santos CG, Moore M, Graybeal B, Washer G. A comparison of nondestructive evaluation methods for bridge deck assessment. NDT \& E International. 2003;36(4):245-255.

[6] Tabatabai H, Tabatabai M, Lee CW. Reliability of bridge decks in Wisconsin. Journal of Bridge Engineering. 2011;16(1):53-62.

[7] Washer G, Nasrollahi M, Applebury C, Connor R, Ciolko A, Kogler R, Fish P, Forsyth D. Proposed guideline for reliability-based bridge inspection practices. NCHRP Rep. 782. 2014.

[8] Reising R. Risk-based bridge inspection practices. [Master degree]. Purdue University, West Lafayette, Indiana. 2014.

[9] Cady PD, Weyers RE. Deterioration rates of concrete bridge decks. Journal of Transportation Engineering. 1984;110(1):34-44.

[10] Nasrollahi M, Washer G. Estimating inspection intervals for bridges based on statistical analysis of national bridge inventory data. Journal of Bridge Engineering. 2015;20(9):04014104.

[11] FHWA. National bridge inspection standards regulations (NBIS). Federal Register. 2004;69(239):15-35.

[12] Everett TD, Weykamp P, Capers Jr HA, Cox WR, Drda TS, Hummel L, Jensen P, Juntunen DA, Kimball T, Washer GA. Bridge evaluation quality assurance in Europe. Rep. No. FHWA-PL-08-016. Federal Highway Administration (FHWA), Washington, DC. 2008.

[13] Mašović S, Hajdin R. Modelling of bridge elements deterioration for Serbian bridge inventory. Structure and Infrastructure Engineering. 2014;10(8):976-987.

[14] Hema J. Concrete bridge deck condition. Prepared for: Utah Department of Transportation Research and Development Division. Submitted by Department of Civil \& Environmental Engineering. 2005.

[15] Nasr A, Björnsson I, Honfi D, Larsson Ivanov O, Johansson J, Kjellström E. A review of the potential impacts of climate change on the safety and performance of bridges. Sustainable and Resilient Infrastructure. 2019:121.

[16] Tolliver D, Lu P. Analysis of bridge deterioration rates: A case study of the northern plains region. Journal of the Transportation Research Forum. 2011; 50(2):87-100.

[17] Bolukbasi M, Mohammadi J, Arditi D. Estimating the future condition of highway bridge components using national bridge inventory data. Practice Periodical on Structural Design and Construction. 2004;9(1):16-25.

[18] Hatami A, Morcous G. Developing deterioration models for Nebraska bridges. Nebraska Department of Roads, Lincoln, NE. 2011.

[19] Wang YZ, He TQ, Liu S, Sun YY. Condition assessment for existing reinforced concrete bridges. In: Earth \& Space 2008: Engineering, Science, Construction, and Operations in Challenging Environments. 2008.p. 18.

[20] Federal Highway Administration (FHWA). Recording and coding guide for the structure inventory and appraisal of the nation's bridges. Rep. No. FHWA-PD-96-001. Washington, DC. 1995.

[21] FHWA. Tables of frequently requested NBI information. U.S. Department of Transportation. 2016.

[22] MDOT. National bridge inventory rating scale. Michigan Department of Transportation, MI. 2015.

[23] White K, Minor J, Derucher KN. Bridge maintenance inspection and evaluation. 2nd Ed. New York: Marcel Dekker, Inc. 1992.

[24] Abdel-Qader I, Yohali S, Abudayyeh O, Yehia S. Segmentation of thermal images for non-destructive evaluation of bridge decks. NDT \& E International. 2008;41(5):395-405.

[25] Abudayyeh O, Yehia S, Abdel-Qader I, Zalt A. GPR imaging for bridge deck condition assessment. Bridge Structures. 2008;4(2):75-86.

[26] Yehia S, Abudayyeh O, Abdel-Qader I, Zalt A. Ground-penetrating radar, chain drag, and ground truth: Correlation of bridge deck assessment data. Transportation Research Record. 2008;2044(1):39-50.

[27] Abudayyeh O, Abdel-Qader I, Nabulsi S, Weber J. Using non-destructive technologies and methods in bridge management systems. Journal of Urban Technology. 2004;11(1):63-76.

[28] ASCE. A comprehensive assessment of America’s infrastructure. https://www.infrastructurereportcard.org/ wp-content/uploads/2016/10/2017-Infrastructure-Report-Card.pdf. (October 2016).

[29] Bhattacharyya H. Kruskal-Wallis test: Theory. USA: Wiley;2014.

[30] Mehrannia H, Pakgohar A. Using easy fit software for goodness-of-fit test and data generation. International Journal of Mathematical. 2014; 5(1):118-124.

[31] Engmann S, Cousineau D. Comparing distributions: the two-sample Anderson-Darling test as an alternative to the Kolmogorov-Smirnoff test. Journal of Applied Quantitative Methods. 2011;6(3):1-17. 
[32] Meeker QW, Escober AL. Statistical methods for reliability data. USA: Wiley; 1998.

[33] Martín J, Pérez CJ. Application of a generalized lognormal distribution to engineering data fitting. Safety, Reliability and Risk Analysis: Theory, Methods and Applications. 2009;1(4): 869-874.

[34] Ginos BF. Parameter estimation for the lognormal distribution. [Master degree]. Brigham Young University, Provo, UT.2009.

[35] Aristizabal RJ. Estimating the parameters of the three-parameter lognormal distribution. [Master degree]. Florida International University, Miami, Florida. 2012.

(C) 2020 by the author(s). This work is licensed under a Creative Commons Attribution 4.0 International License (http://creativecommons.org/licenses/by/4.0/). Authors retain copyright of their work, with first publication rights granted to Tech Reviews Ltd. 